\title{
Important Transfer Power Lines Analysis under Large Scale Direct Electricity Purchases
}

\author{
Lili Liu ${ }^{1,}$, Jie $\mathrm{Yu}^{1, b^{*}}$ and Bike Xue ${ }^{2, \mathrm{c}}$ \\ ${ }^{1}$ School of Electrical Engineering, Southeast University, Nanjing, Jiangsu Province, China \\ ${ }^{2}$ China Electric Power Research Institute, Nanjing, Jiangsu Province, China \\ a15195769753@163.com, byujie@seu.edu.cn, xuebike@epri.sgcc.com.cn
}

Keywords: Large consumers direct purchase of electricity; Breadth first search; Sensitivity analysis; Transmission path

\begin{abstract}
Large direct power consumers not only can stimulate the power market potential but also can be a breakthrough in the reform of the market. The Research is about transmission path of the direct power purchase, which supports the stability analysis of power grid operation and branch block management. This paper will propose a sensitivity analysis method based on breadth first search, the breadth first search method can search out all transmission paths, the combination of the breadth first search method and the sensitivity analysis can recreate the superposed sensitivity. For this purpose IEEE 118 bus system has been used as example and the results will show how useful and effective this method can be.
\end{abstract}

\section{Introduction}

Large consumers and the power company will sign a two-sided power-purchasing contract to carry out this direct purchase of power. This will benefit the large consumers to cut their prices and they can also reduce the production cost as well as to improve the competence of the company and adjust supply-demand relationship with the attendance of power enterprise. Power companies delivers electricity to the client through grid. For this purpose, an analysis of transmission path should be made to analyze the effect of this electricity trade on the stability of the power branch and grid respectively [1-5].

To calculate the transmission path, power load flow tracing method [6-9] is usually applied. But, in this method the calculation is based on the share of delivering cost, and the transmission cost depends on the market standards and other factors. Another way electrical dissection method [10-13] can compute the transmission route from the power company to client, based on specific physical parameters of grid. But computing results include all the path of supply network, which makes it difficult to determine the effect of large consumers direct-purchasing on the branch from which the power is to be supplied.

To solve the above mentioned issue, this article will introduce sensitivity analysis study of breadth first search method to analyze the transmission path of the direct-purchasing trade by large consumers. According to trade information and grid structure, sensitivity analysis method combined with breadth first search is applied to search all the possible transmission paths of more than one large consumers of direct-purchasing of electricity. It will also help to determine the superimposed sensitivity of the path and its order, combined with the computing results of sensitivity.

\section{Sensitivity Analysis Principle}

Based on the system power flow equation and the qualitative physical concept, sensitivity analysis method [14] obtains the sensitivity of the dependent variable to the independent variable by using the changes of some physical variables in the system.

The power flow of the transmission line, which is caused by the power change of the generator node, is known as the sensitivity of the power of the generator node to the branch power flow. This sensitivity [15] defines the influence of the node power change on the transmission power. 
When the power transfer of the direct electricity purchase nodes between $\mathrm{i}$ and $\mathrm{I}$ change $\Delta P_{i j}$, namely active power injection of node $\mathrm{i}$ and node $\mathrm{j}$ change respectively $+\Delta P_{i j}$ and $-\Delta P_{i j}$, the active power injection of other nodes unchanged, that is, the change of the active power injection amount of node $\mathbf{i}$ and node $\mathbf{I}$ is equal and in the opposite direction.

Current changes of branch $\mathrm{k}$ obtained by sensitivity calculations [15]:

$\Delta P_{k}^{i j}=\frac{1}{x_{k}} M_{k}^{T} \mathrm{X}\left[e_{i} \vdots e_{j}\right]\left[\begin{array}{c}1 \\ -1\end{array}\right] \Delta P_{i j}=\frac{x_{m i}-X_{m j}-X_{n i}+X_{n j}}{x_{k}} \Delta P_{i j}$

which is:

$\left\{\begin{array}{c}\Delta P_{k}^{i j}=S_{k-i j} \Delta P_{i j} \\ S_{k-i j}=\frac{X_{m i}-X_{m j}-X_{n i}+X_{n j}}{x_{k}}\end{array}\right.$

where is:

$\Delta P_{i j}$ denotes the active power injection change of direct purchase of electricity at node $\mathrm{i}$ and node j;

$\Delta P_{k}^{i j}$ means current changes in branch $\mathrm{k}$;

$S_{k-i j}$ is the sensitivity of direct electricity purchase at node $\mathbf{i}, \mathbf{j}$ on branch $\mathrm{k}$;

$x_{k}$ represents reactance of branch $\mathrm{k}$;

$\mathrm{X}$ is the inverse matrix of $B_{0}$ in DC power flow, $B_{0}$ is $\mathrm{n} \times \mathrm{n}$ matrix with admittance of branch created, $X_{m i}$ is the $m t h$ row and ith column element in matrix $\mathrm{X}, X_{m j}$ is the $m$ th row and $j$ th column element in matrix $\mathrm{X}, X_{n i}$ is the nth row ith column element in matrix $\mathrm{X}$, $X_{n j}$ is the nth row and $\mathrm{jth}$ column element in matrix $\mathrm{X}$;

$M_{k}$ is the correlation matrix of branch $\mathrm{k}$ (starting node $\mathrm{m}$ and ending node $\mathrm{n}$ ), $M_{k}=\frac{[0 \cdots 1 \cdots-1 \cdots 0]^{T}}{m}$

$e_{i}$ is column vector as a unit, only node i corresponding to the position of non-zero elements and rest of the elements are zero;

$e_{j}$ is column vector as a unit, only node $\mathbf{j}$ corresponding to the position of non-zero elements and rest of the elements are zero.

\section{Combined BFS Sensitivity Analysis}

The mean of direct purchase is a kind of agreement between large electricity consumers and power plants. In this paper, the important power transmission path is studied under direct purchase agreement. The influence degree of transmission lines is analyzed based on sensitivity analysis method. Then, the breadth first search (BFS) method is used to do traversal and sorting of the important transmission line. 
Breadth First Search Theory. Breadth first search belong to one of the basic methods of graph theory search. Search graph refers to a vertex from the rest of the search graph vertices, and each vertex is accessed only once [16].

Breadth first search follow principle is "neighboring nodes to be accessed first" prior to "adjacent nodes to be accessed", the basic idea is [17]:

1) First access the specified starting node $N_{s t}$ and mark it as accessed;

2) Then from node $N_{s t}$ starts to connect the adjacent nodes, referred to as a first-tier node $N_{11}$, $N_{12}, \ldots . .$, and mark them as accessed;

3) Then follow $N_{11}, N_{12}, \cdots \cdots$ order to access all the adjacent nodes, referred to as second-tier nodes $N_{21}, N_{22}, \cdots \cdots$, and are mark as accessed;

4) Next step is to start over again to make sure that every node has been accessed, and marked;

5) It continues, until access to the termination node $N_{\text {end }}$.

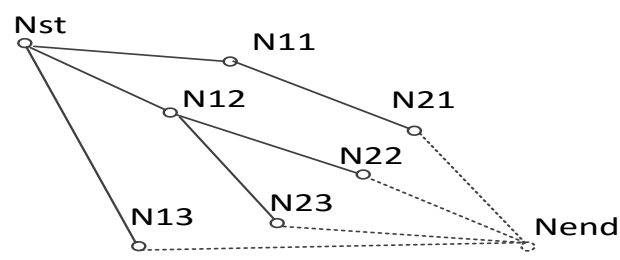

Figure 1. Breadth first search schematic

Combined BFS Sensitivity Analysis. In this paper, combination of breadth first search sensitivity analysis of large consumers of direct purchase of electricity trading in the transmission path analysis is used. Use breadth first search algorithm to search out all the possible transmission path. And combined with sensitivity calculations, a sorted form of superimposed sensitivity can be selected.

Breadth first search method to search out all the transmission paths from the direct purchase of electricity trading between the generator node $\mathrm{i}\left(N_{s t}\right)$ and large user node $\mathrm{j}\left(N_{e n d}\right)$, the combined sensitivity calculation, which is:

1) From the generator node i start search to adjacent node $N_{11}, N_{12}, \ldots$, mark as accessed and calculate the sensitivity $S_{N_{s t}-N_{11}}, S_{N_{s t}-N_{12}}, \ldots$;

2)After this search for 1) the $N_{11}, N_{12}, \cdots \cdots$ not accessed adjacent node $N_{21}, N_{22}, \ldots$, marked as accessed, and calculate sensitivity $S_{N_{s t}-N_{21}}, S_{N_{s t}-N_{22}}, \ldots$;

3) And so on until the search to large user node $\mathbf{j}$.

By Eq. 2 it can be drawn from $\mathrm{k}$ (starting and ending node i.e. $\mathrm{m}, \mathrm{n}$ ), the sensitivity is:

$S_{k-N_{s t} N_{\text {end }}}=\frac{x_{m N_{s t}}-X_{m N_{E n d}}-X_{n N_{s t}}+x_{n N_{e n d}}}{x_{k}}$

When $\mathbf{R}$ exist large consumers direct purchase of electricity trading, the $r$ transactions(transaction beginning and ending node i.e. $N_{r s t}, N_{\text {rend }}$ ) The sensitivity of the branch $\mathrm{k}$ :

$S_{k-N_{r s t} N_{\text {rend }}}=\frac{x_{m N_{r s t}}-X_{m N_{\text {rend }}}-X_{n N_{r s t}}+X_{n N_{\text {rend }}}}{x_{k}} \mathrm{r}=1,2, \ldots \ldots, \mathrm{R}$

Branch $\mathrm{k}$ superimposed sensitivity:

$S_{\text {sum }}=\sum_{r=1}^{R} S_{k-N r_{\text {st }} N_{\text {rend }}}$

A flowchart of the algorithm is shown in Fig. 2. When more than one consumers trading co-exist. 


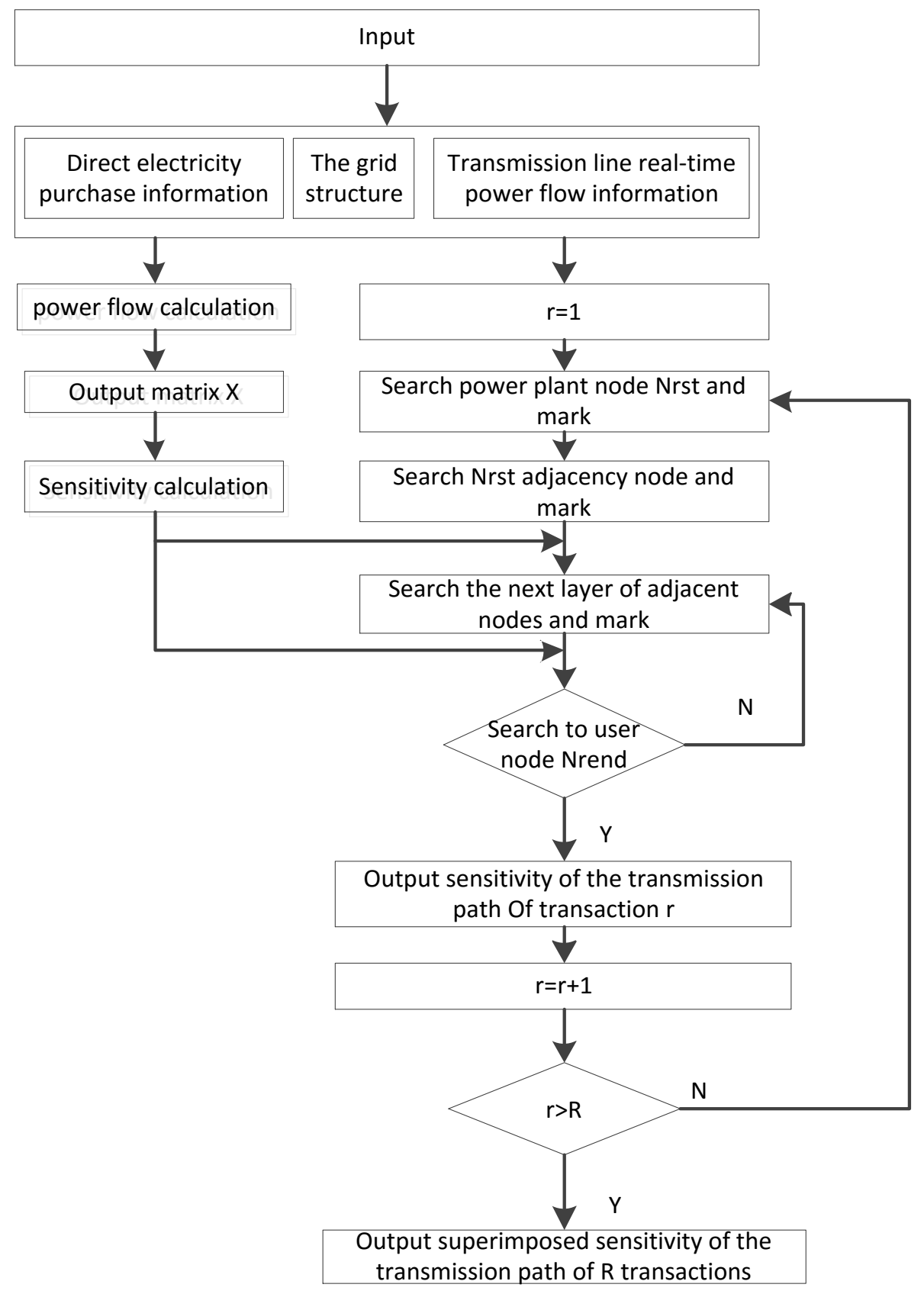

Figure 2. Direct Power Purchase Contract Transmission path flowchart

\section{Examples}

In order to verify the proposed method is reasonable and effective, IEEE 118-bus system is tested.

In this example four large scale consumers of direct purchase of electricity is considered. For this purpose breadth first search method in the IEEE bus system has been used. In IEEE 118 bus system, the direct purchase of electricity trading 1 is marked with red color. The transaction end nodes are node 27 and node 60 . The 2 nd direct purchase is marked with green color. The transaction end nodes are node 5 and node 65 . The 3rd direct purchase is marked with blue color. The transaction end nodes are node 8 and node 79. The 4th direct purchase is marked with orange color. The transaction end node are node 2 and node 77. Utility transmission channel is marked with purple color. As shown in Fig. 3: 


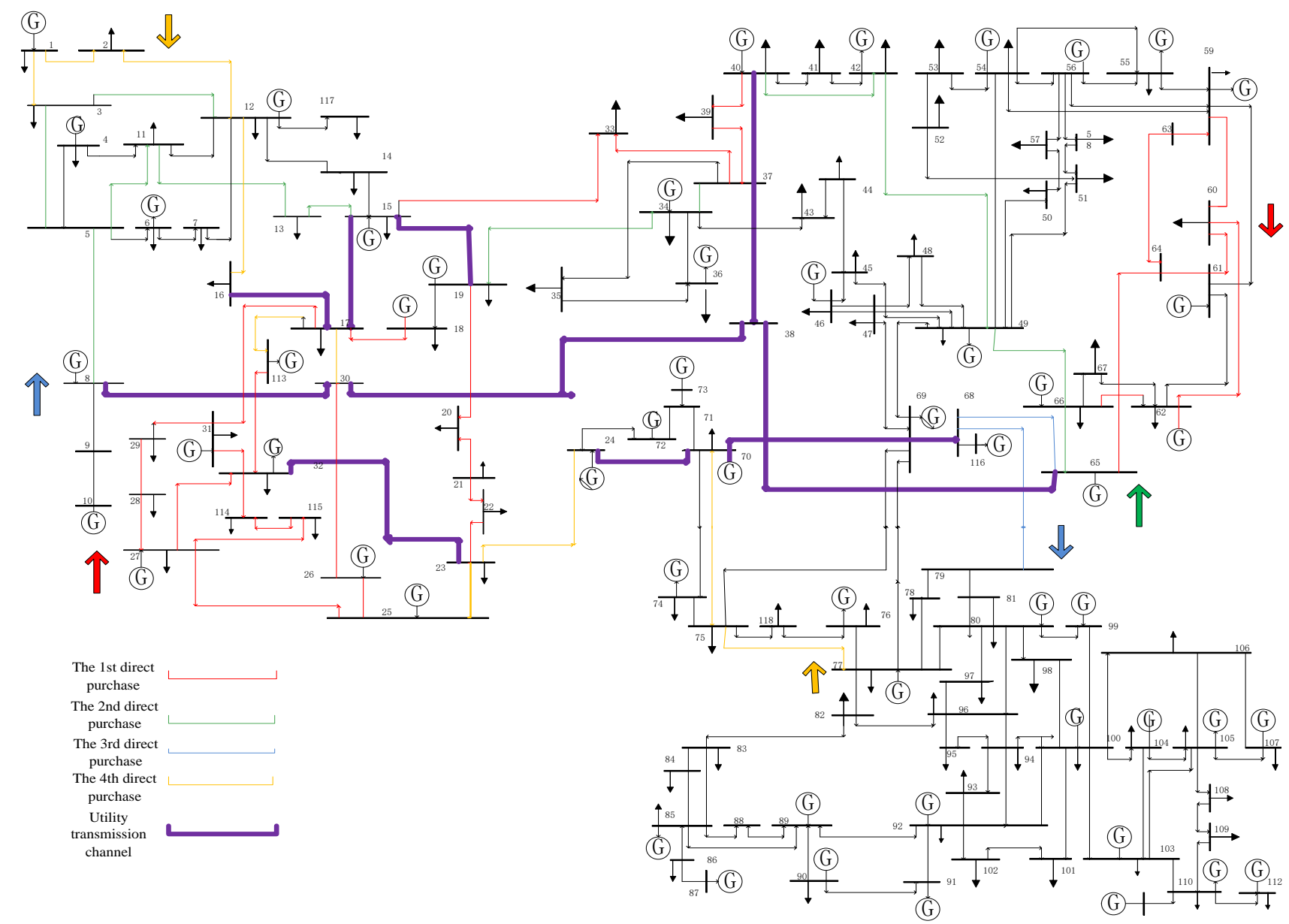

Figure 3. Direct power purchase transmission roadmap

Then calculate the sensitivity and superimposed branch sensitivity according to the sensitivity formula and then analysis of the impact of large scale branch direct purchase of electricity trading are performed simultaneously and are sorted based on the sensitivity importance.

Based on the sensitivity analysis, the transmission path of direct purchase of electricity can be calculated. Combined with the direct trade transmission path showed in Fig. 3, the accumulated sensitivity of the important transmission paths can be obtained, and can be sorted in descending form as shown in Table 1:

Table 1 Transmission path superimposed sensitivity table

\begin{tabular}{|l|l|l|}
\hline $\begin{array}{l}\text { Sensitivity } \\
\text { ranking }\end{array}$ & $\begin{array}{l}\text { Branch } \\
\text { node }\end{array}$ & Branch node \\
\hline 1 & 30 & 38 \\
2 & 38 & 65 \\
3 & 8 & 30 \\
4 & 24 & 70 \\
5 & 23 & 32 \\
6 & 70 & 69 \\
7 & 17 & 15 \\
8 & 68 & 69 \\
9 & 37 & 40 \\
10 & 15 & 19 \\
11 & 38 & 37 \\
12 & 16 & 17 \\
\hline
\end{tabular}




\section{Analysis Summary}

According to the method proposed in this paper, the superimposed sensitivity of multiple transactions transmission paths can be obtained. When several transactions go through the same branch, superimposed sensitivity is applied to the branch to analyze the influence degree of transactions based on the sensitivity calculation results. Based on the IEEE 118 bus system as an example for example analysis, the results show the rationality and validity of the proposed method.

\section{References}

[1] Q. Xia, Y. Bai, H.W. Zhong and Q.X. Chen: Automation of Electric Power Systems, Vol. 37 (2013) No.20, p.1. (In Chinese)

[2] G.H. Chen, L.Q. Lin, B.Y. Wen and Y.W. Jiang: GUANGDONG ELECTRIC POWER, Vol. 25 (2012) No.6, p.19. (In Chinese)

[3] S.L. Zhang, Y. Zhang and H.Y. Chen: East China Electric Power, Vol. 37 (2009) No.4, p.523. (In Chinese)

[4] H.Y. Chen, S.L. Zhang and Y. Zhang: Power System Technology, Vol. 32 (2008) No.21, p.85. (In Chinese)

[5] J.Y. Hu and X.Y. Chen: Power System Technology, Vol. 31 (2007) No.24, p.40. (In Chinese)

[6] B. J: IEE Proceedings of Generation, Transmission and Distribution, Vol. 143 (1996) No.4, p.313.

[7] K. D, A. R and S. G: IEEE Transactions on Power Systems, Vol. 12 (1997) No.1, p.52.

[8] C.Y. Li. Model and Algorithm of power Flow Tracing and its Application in Transmission Congestion Management (ph. D., Chongqing University, China2008), p.4. (In Chinese)

[9] L. Ma, K.G. Xie, J.Q. Zhou, P. Zhou, Y. Zhao and X. Zhao: Journal of Chongqing University, Vol. 27 (2004) No.7, p.45. (In Chinese)

[10]J.L. Yu and Z. Liu: Automation of Electric Power Systems, Vol. 29 (2005) No.1, p.22. (In Chinese)

[11]Y. Tang, F. Yuan, X. Zhou and Q.L. Wan: Automation of Electric Power Systems, Vol. 31 (2007) No.19, p.14. (In Chinese)

[12]W.S. Tian and J.L. Yu: Power System Technology, Vol. 34 (2010) No.3, p.124. (In Chinese)

[13]J.L. Yu and Y. Tang: Proceedings of the CSEE, Vol. 27 (2007) No.16, p.38. (In Chinese)

[14]J. Luo: Introduction to System Sensitivity Theory (NORTHWESTERN POLYTECHNICAL UNIVERSITY PRESS, China 1990), p.16. (In Chinese)

[15]B.M. Zhang, S.S. Chen and Z. Yan: The Higher Power Network Analysis (TSINGHUA UNIVERSITY PRESS, China 2007), p.202. (In Chinese)

[16]J.F. Tian and X.J. Lei: PR\&AI, Vol. 25 (2012) No.3, p.481. (In Chinese)

[17]Z.M. Yang: Agriculture Network Information, (2009) No.12, p.136. (In Chinese) 